\title{
Continuous Reformulations for Zero-One Programming Problems
}

\author{
M. De Santis • F. Rinaldi
}

Received: 21 January 2011 / Accepted: 17 September 2011

(C) Springer Science+Business Media, LLC 2011

\begin{abstract}
In this work, we study continuous reformulations of zero-one programming problems. We prove that, under suitable conditions, the optimal solutions of a zero-one programming problem can be obtained by solving a specific continuous problem.
\end{abstract}

Keywords Zero-one programming · Concave functions · Continuous programming

\section{Introduction}

Several important problems arising in operations research, graph theory and mathematical programming are formulated as $0-1$ programming problems. A possible approach for solving this class of problems can be that of transforming the original problem into an equivalent continuous problem. Various transformations have been proposed in the literature (see, e.g., [1-6]). A well-known continuous reformulation comes out by relaxing the integrality constraints on the variables and by adding a penalty term to the objective function. This approach has been first introduced by Raghavachari [7] to solve 0-1 linear programming problems, then extended by Giannessi and Niccolucci [8] to general nonlinear integer programming problems. Many other reformulations related to the one by Raghavachari have been proposed in the literature (see, e.g., [9-14]). An interesting analysis of continuous penalty reformulations for integer programming problems has been given in [15].

\footnotetext{
M. De Santis · F. Rinaldi ( $₫)$

Dipartimento di Informatica e Sistemistica "Antonio Ruberti”, Sapienza University of Rome, Rome, Italy

e-mail: rinaldi@dis.uniroma1.it

M. De Santis

e-mail: mdesantis@dis.uniroma1.it
} 
In this paper, we propose a different continuous reformulation for solving 0 -1 programming problems obtained by relaxing the integrality constraints on the variables and by making a nonlinear transformation of the variables in the objective function. It can be proved that, under suitable assumptions, a given binary problem and its continuous reformulation are equivalent. The paper is organized as follows. In Sect. 2, we show a general equivalence result between a $0-1$ programming problem and a continuous problem. In Sect. 3, we define various continuous reformulations, and we show (using the general results stated in Sect. 2) that a binary problem and its continuous reformulations share the same global minima. Finally, in Sect. 4, we draw some conclusions.

\section{Equivalent Continuous Reformulations for Zero-One Programming Problems}

We start from the zero-one programming problem

$$
\min c^{T} x \quad \text { s.t. } \quad x \in C, \quad x \in\{0,1\}^{n},
$$

where $C \subset \mathbb{R}^{n}$ is a convex set.

Then, we consider the following nonlinear constrained problem

$$
\min f(x) \quad \text { s.t. } \quad x \in C, \quad 0 \leq x \leq e,
$$

where

$$
f(x):=\sum_{\substack{i=1 \\ c_{i}>0}}^{n} c_{i} g_{i}\left(x_{i}\right)+\sum_{\substack{i=1 \\ c_{i}<0}}^{n}\left|c_{i}\right| g_{i}\left(x_{i}\right)+\sum_{\substack{i=1 \\ c_{i}=0}}^{n} g_{i}\left(x_{i}\right)-\sum_{\substack{i=1 \\ c_{i}<0}}^{n}\left|c_{i}\right|,
$$

and $g_{i}:[0,1] \rightarrow \mathbb{R}, i=1, \ldots, n$, are continuous and concave functions. We assume, without any loss of generality, that there exists at least an extreme point $y$ of the feasible set related to (CP) such that $y_{i} \notin\{0,1\}$ for some index $i=1, \ldots, n$.

In order to prove the equivalence between Problem (IP) and Problem (CP), we make some assumptions on the set of extreme points of (CP) and on the functions $g_{i}$ used in the definition of $f$. Let $S \subset[0,1]^{n}$ be the set of extreme points of (CP). We define two values $x_{l}$ and $x_{u}$ as follows:

$$
x_{l}:=\inf _{x \in S} l(x), \quad x_{u}:=\sup _{x \in S} u(x)
$$

where

$$
l(x):= \begin{cases}\min \left\{x_{i}: i=1, \ldots, n ; x_{i} \neq 0\right\} & \text { if } x \neq 0 \\ 1 & \text { if } x=0\end{cases}
$$




$$
u(x):= \begin{cases}\max \left\{x_{i}: i=1, \ldots, n ; x_{i} \neq 1\right\} & \text { if } x \neq e \\ 0 & \text { if } x=e\end{cases}
$$

Then, for any component $x_{i} \notin\{0,1\}$ of a point $x \in S$ we have that

$$
x_{i} \in\left[x_{l}, x_{u}\right] .
$$

Assumption 2.1 For the feasible set of problem (CP) there exists a value $\epsilon>0$ such that $x_{l}>\epsilon$ and $1-x_{u}>\epsilon$.

This is equivalent to saying that $x_{l} \neq 0$ and $x_{u} \neq 1$. In Fig. 1, we have two examples of convex feasible sets of (CP) satisfying Assumption 2.1.

Assumption 2.2 For all indices $i$ such that $c_{i}>0$, we have

(i) $g_{i}(0)=0, g_{i}(1)=1$;

(ii) $g_{i}\left(x_{i}\right)>\frac{(n+1) \max _{i}\left|c_{i}\right|+\sum_{i}\left|c_{i}\right|}{\min _{i}\left|c_{i}\right|}$ if $x_{i} \in\left[x_{l}, x_{u}\right]$.

For all indices $i$ such that $c_{i}<0$, we have

(iii) $g_{i}(0)=1, g_{i}(1)=0$;

(iv) $g_{i}\left(x_{i}\right)>\frac{(n+1) \max _{i}\left|c_{i}\right|+\sum_{i}\left|c_{i}\right|}{\min _{i}\left|c_{i}\right|}$ if $x_{i} \in\left[x_{l}, x_{u}\right]$.

For all indices $i$ such that $c_{i}=0$, we have

(v) $g_{i}(0)=0, g_{i}(1)=0$;

(vi) $g_{i}\left(x_{i}\right)>n \max _{i}\left|c_{i}\right|+\sum_{i}\left|c_{i}\right|$ if $x_{i} \in\left[x_{l}, x_{u}\right]$.

We report here some important results about the minimization of a concave function over a closed and convex set (see [16] for further details):
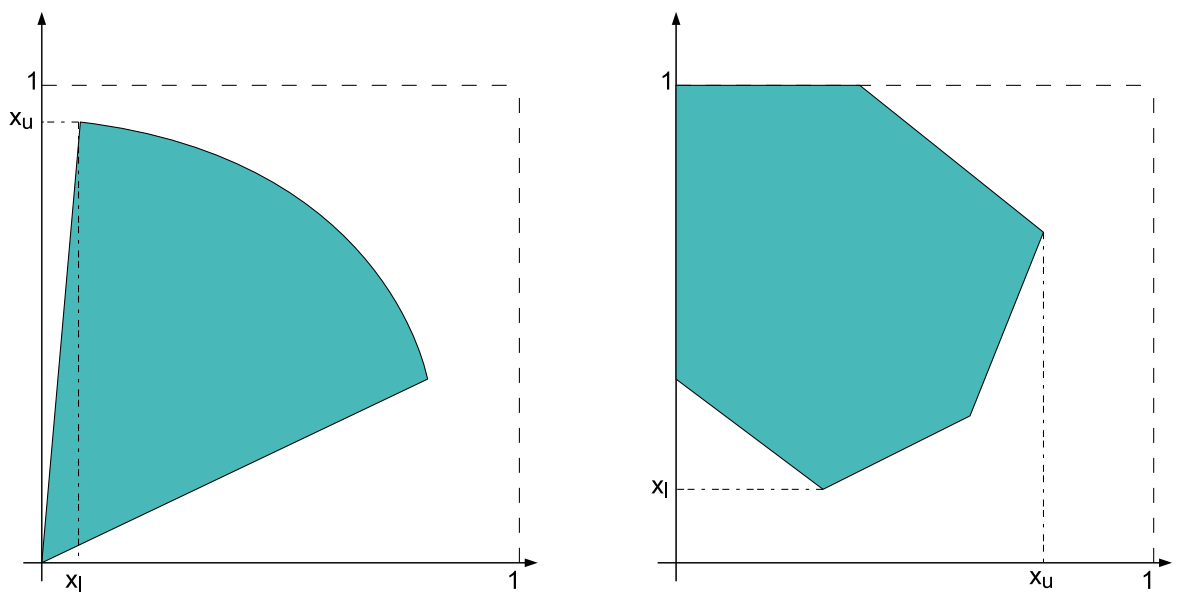

Fig. 1 Examples of feasible sets satisfying Assumption 2.1 
Proposition 2.1 Let $f$ be a concave function, and let $C$ be a closed and convex set contained in dom $f$. Suppose that $C$ contains no lines. If the infimum of $f$ relative to $C$ is attained at all, then it is attained at some extreme points of $C$.

Proposition 2.2 Let $f$ be a concave function, and let $C$ be a nonempty polyhedral convex set contained in dom $f$. Suppose that $C$ contains no lines, and that $f$ is bounded from below on $C$. Then the infimum of $f$ relative to $C$ is attained at one of the (finitely many) extreme points of $C$.

Now, we can prove the equivalence between the zero-one programming problem (IP) and its continuous concave reformulation (CP).

Theorem 2.1 If Assumptions 2.1 and 2.2 hold, then problems (IP) and (CP) have the same minimum points.

Proof We first prove that if $x^{*}$ is a solution of (IP) then $x^{*}$ is a solution of (CP). Let $x^{*}$ be a solution of (IP) and suppose by contradiction that there exists a point $\bar{x}$ which is the solution of (CP) such that

$$
f(\bar{x})<f\left(x^{*}\right)=c^{T} x^{*} .
$$

We consider three cases:

1. Suppose that $\bar{x}_{i} \in\{0,1\}$ for all $i=1, \ldots, n$ :

$$
c^{T} \bar{x}=f(\bar{x})<f\left(x^{*}\right)=c^{T} x^{*} .
$$

This cannot be the case as there would exists $\bar{x} \in C \cap\{0,1\}^{n}$ such that $c^{T} \bar{x}<$ $c^{T} x^{*}$, contradicting the fact that $x^{*}$ is the optimum of (IP).

2. Suppose that $\exists j \in\{1, \ldots, n\}$ s.t. $\bar{x}_{j} \notin\{0,1\}$ :

If $c_{j}>0$, by (i) and (ii) in Assumption 2.2, we have:

$$
f(\bar{x})=\sum_{i: \bar{x}_{i}=1} c_{i}+c_{j} g_{j}\left(\bar{x}_{j}\right) \geq-\sum_{i}\left|c_{i}\right|+\min _{i}\left|c_{i}\right| g_{j}\left(\bar{x}_{j}\right)>n \max _{i}\left|c_{i}\right| .
$$

If $c_{j}<0$, by (iii) and (iv) in Assumption 2.2, we have:

$$
\begin{aligned}
f(\bar{x}) & =\sum_{i: \bar{x}_{i}=1} c_{i}+\left|c_{j}\right| g_{j}\left(\bar{x}_{j}\right)-\left|c_{j}\right| \\
& \geq-\sum_{i}\left|c_{i}\right|+\min _{i}\left|c_{i}\right| g_{j}\left(\bar{x}_{j}\right)-\max _{i}\left|c_{i}\right|>n \max _{i}\left|c_{i}\right| .
\end{aligned}
$$

Finally, if $c_{j}=0$, by (v) and (vi) in Assumption 2.2, we have:

$$
f(\bar{x})=\sum_{i: \bar{x}_{i}=1} c_{i}+g_{j}\left(\bar{x}_{j}\right) \geq-\sum_{i}\left|c_{i}\right|+g_{j}\left(\bar{x}_{j}\right)>n \max _{i}\left|c_{i}\right| .
$$

Hence, for each $x \in C \cap\{0,1\}^{n}$ we have

$$
f(\bar{x})>n \max _{i}\left|c_{i}\right|>c^{T} x
$$


which implies

$$
f(\bar{x})>c^{T} x^{*},
$$

but this contradicts (4).

3. Suppose that $\exists J \subseteq\{1, \ldots, n\}$ s.t. for all $j \in J, \bar{x}_{j} \notin\{0,1\}$ :

By using a similar reasoning as in the proof of case 2 , we have

$$
f(\bar{x})>c^{T} x^{*}
$$

but this contradicts (4).

We now prove that if $\bar{x}$ is a solution of (CP) then $\bar{x}$ is a solution of (IP).

If $\bar{x}$ is a solution of $(\mathrm{CP})$ then, since we are minimizing a concave function over a compact and convex set, by Proposition 2.1, we have that $\bar{x}$ is an extreme point of $C \cap[0,1]^{n}$.

We first prove that $\bar{x} \in\{0,1\}^{n}$.

By contradiction, we suppose that there exists an index $j \in\{1, \ldots, n\}$ such that $\bar{x}_{j} \notin\{0,1\}$. By repeating the same arguments used in the first part of the proof (case 2), for all $x \in C \cap\{0,1\}^{n}$ we have

$$
f(\bar{x})>c^{T} x=f(x),
$$

thus obtaining a contradiction.

Now, suppose by contradiction that there exists $x^{*}$, a solution of (IP), such that

$$
c^{T} x^{*}<c^{T} \bar{x} .
$$

Since $\bar{x} \in\{0,1\}^{n}$, we have that $f(\bar{x})=c^{T} \bar{x}$, thus (6) implies that $f\left(x^{*}\right)<f(\bar{x})$, contradicting the optimality of $\bar{x}$ for $(\mathrm{CP})$. The theorem is then proved.

We can apply the previous result to the case of zero-one linear programming problems. Suppose that we are dealing with the following problem:

$$
\min c^{T} x \quad \text { s.t. } \quad x \in P, \quad x \in\{0,1\}^{n},
$$

where $P$ is a polyhedral set. Then we can prove the equivalence of (ILP) with the following problem

$$
\min f(x) \quad \text { s.t. } \quad x \in P, \quad 0 \leq x \leq e,
$$

where the function $f:[0,1]^{n} \rightarrow \mathbb{R}$ is defined as in (1). The following result is a straightforward application of Theorem 2.1.

Proposition 2.3 If Assumption 2.2 holds, then problems (ILP) and (LP) have the same minimum points. 
Proof First of all, we see that the feasible set of problem (LP) satisfies Assumption 2.1. Let $V$ be the set of the vertices of the polyhedron $P$. Since the cardinality of $V$ is finite, we have

$$
x_{l}=\min _{x \in V} l(x), \quad x_{u}=\max _{x \in V} u(x),
$$

with $l(x)$ and $u(x)$ defined as in (2) and (3). It is easy to see that there exists a value $\epsilon>0$ such that $x_{l}>\epsilon$ and $1-x_{u}>\epsilon$. In other words, $x_{l}$ and $x_{u}$ are respectively the minimum and the maximum values (different from 0 and 1) that can be assumed by the components of the vertices in $P$.

The rest of the proof is a verbatim repetition of Theorem 2.1 .

We remark that if the feasible set of the continuous reformulation is polyhedral, Assumption 2.1 is always satisfied.

\section{Examples of Continuous Reformulations}

In this section, starting from the ideas developed in [17], we propose various examples of continuous reformulations for solving a given zero-one programming problem, and we show (using the general results stated in the previous section) that these reformulations have the same global minimizers of the original zero-one programming problem.

First of all, we denote

$$
\tilde{c}:=\frac{(n+1) \max _{i}\left|c_{i}\right|+\sum_{i}\left|c_{i}\right|}{\min _{i}\left|c_{i}\right|} .
$$

Now we can define the functions $g_{i}$ to be used in (1):

Case $c_{i}>0$ :

$$
\begin{aligned}
& g_{i}(t):=\min \left\{\gamma_{1_{+}} \phi(t), 1+\gamma_{2_{+}} \phi(1-t)\right\}, \\
& \gamma_{1_{+}}>\frac{\tilde{c}}{\phi\left(x_{l}\right)}, \quad \gamma_{2_{+}}>\frac{\tilde{c}-1}{\phi\left(1-x_{u}\right)}
\end{aligned}
$$

Case $c_{i}<0$ :

$$
\begin{aligned}
& g_{i}(t):=\min \left\{1+\gamma_{1_{-}} \phi(t), \gamma_{2_{-}} \phi(1-t)\right\}, \\
& \gamma_{1_{-}}>\frac{\tilde{c}-1}{\phi\left(x_{l}\right)}, \quad \gamma_{2_{-}}>\frac{\tilde{c}}{\phi\left(1-x_{u}\right)}
\end{aligned}
$$

Case $c_{i}=0$ :

$$
\begin{aligned}
& g_{i}(t):=\min \left\{\gamma_{0} \phi(t), \gamma_{0} \phi(1-t)\right\}, \\
& \gamma_{0}>\max \left\{\frac{n \max _{i}\left|c_{i}\right|+\sum_{i}\left|c_{i}\right|}{\phi\left(x_{l}\right)}, \frac{n \max _{i}\left|c_{i}\right|+\sum_{i}\left|c_{i}\right|}{\phi\left(1-x_{u}\right)}\right\} ;
\end{aligned}
$$

with $\phi: \mathbb{R} \rightarrow \mathbb{R}$ a strictly increasing and concave function such that $\phi(0)=0$. 
By choosing functions $g_{i}$ equal to (7), (9) and (11), we can prove that problems (IP) and (CP) are equivalent.

Proposition 3.1 If Assumption 2.1 holds and functions $g_{i}$ are defined as in (7), (9) and (11), then problems (IP) and (CP) have the same minimum points.

Proof We only need to prove that the functions $g_{i}$ used in the definition of the objective function of the continuous reformulation, satisfy Assumption 2.2.

Since $\tilde{c}>1$ and

$$
\epsilon<x_{l} \leq x_{u}<1-\epsilon,
$$

we have that all the $\gamma$-parameters are strictly greater than zero.

We consider three different cases:

1. $c_{i}>0$ : the choice of the parameters $\gamma_{1_{+}}$and $\gamma_{2_{+}}$, and the fact that $\tilde{c}>1$ guarantee,

$$
g_{i}(0)=\min \left\{0,1+\gamma_{2_{+}} \phi(1)\right\}=0
$$

and

$$
g_{i}(1)=\min \left\{\gamma_{1_{+}} \phi(1), 1\right\}=1 .
$$

Furthermore, for all $x_{i} \in\left[x_{l}, x_{u}\right]$, we have

$$
\begin{aligned}
g_{i}\left(x_{i}\right) & =\min \left\{\gamma_{1_{+}} \phi\left(x_{i}\right), 1+\gamma_{2_{+}} \phi\left(1-x_{i}\right)\right\} \\
& >\min \left\{\frac{\tilde{c} \phi\left(x_{i}\right)}{\phi\left(x_{l}\right)}, 1+\frac{(\tilde{c}-1) \phi\left(1-x_{i}\right)}{\phi\left(1-x_{u}\right)}\right\}>\tilde{c} .
\end{aligned}
$$

2. $c_{i}<0$ : the choice of the parameters $\gamma_{1_{-}}$and $\gamma_{2_{-}}$, and the fact that $\tilde{c}>1$ guarantee,

$$
g_{i}(0)=\min \left\{1, \gamma_{2_{-}} \phi(1)\right\}=1
$$

and

$$
g_{i}(1)=\min \left\{1+\gamma_{1_{-}} \phi(1), 0\right\}=0 .
$$

Furthermore, for all $x_{i} \in\left[x_{l}, x_{u}\right]$, we have

$$
\begin{aligned}
g_{i}\left(x_{i}\right) & =\min \left\{1+\gamma_{1_{-}} \phi\left(x_{i}\right), \gamma_{2_{-}} \phi\left(x_{i}\right)\right\} \\
& >\min \left\{1+\frac{(\tilde{c}-1) \phi\left(x_{i}\right)}{\phi\left(x_{l}\right)}, \frac{\tilde{c} \phi\left(1-x_{i}\right)}{\phi\left(1-x_{u}\right)}\right\}>\tilde{c} .
\end{aligned}
$$

3. $c_{i}=0$ : the choice of the parameter $\gamma_{0}$ guarantees

$$
g_{i}(0)=\min \left\{0, \gamma_{0} \phi(1)\right\}=\min \left\{\gamma_{0} \phi(1), 0\right\}=g_{i}(1)=0 .
$$

Furthermore, for all $x_{i} \in\left[x_{l}, x_{u}\right]$, we have

$$
g_{i}\left(x_{i}\right)=\min \left\{\gamma_{0} \phi\left(x_{i}\right), \gamma_{0} \phi\left(1-x_{i}\right)\right\}>n \max _{i}\left|c_{i}\right|+\sum_{i}\left|c_{i}\right| .
$$

Then Assumption 2.2 is satisfied. 
The following result is obtained as an immediate consequence of Proposition 2.3:

Corollary 3.1 If functions $g_{i}$ are defined as in (7), (9) and (11), then problems (ILP) and (LP) have the same minimum points.

We give here some examples of $\phi$ functions:

\section{Exponential Function}

$$
\phi(t)=1-e^{-\alpha t}
$$

with $\alpha>0$;

\section{Logistic function}

$$
\phi(t)=\frac{1-e^{-\alpha t}}{2\left(1+e^{-\alpha t}\right)}
$$

with $\alpha>0$;

\section{Logarithmic function}

$$
\phi(t)=\ln (t+\epsilon)-\ln \epsilon
$$

with $\epsilon>0$;

\section{Hyperbolic functions}

$$
\phi(t)=-(t+\epsilon)^{-p}+\epsilon^{-p}
$$

with $\epsilon>0$.

By setting the functions $\phi$ equal to the exponential term (14), we can define the objective function of the continuous problem (CP) as follows:

$$
\begin{aligned}
& f(x)= \sum_{\substack{i=1 \\
c_{i}>0}}^{n} c_{i} \min \left\{\gamma_{1_{+}}\left(1-e^{-\alpha x_{i}}\right), 1+\gamma_{2_{+}}\left(1-e^{-\alpha\left(1-x_{i}\right)}\right)\right\} \\
&+\sum_{\substack{i=1 \\
c_{i}<0}}^{n}\left|c_{i}\right| \min \left\{1+\gamma_{1_{-}}\left(1-e^{-\alpha x_{i}}\right), \gamma_{2_{-}}\left(1-e^{-\alpha\left(1-x_{i}\right)}\right)\right\} \\
&+\sum_{\substack{i=1 \\
c_{i}=0}}^{n} \min \left\{\gamma_{0}\left(1-e^{-\alpha x_{i}}\right), \gamma_{0}\left(1-e^{-\alpha\left(1-x_{i}\right)}\right)\right\}-\sum_{i=1}^{n}\left|c_{i}\right| . \\
& c_{i}<0
\end{aligned}
$$

In Fig. 2, we report the various functions that can be used in the reformulation of a zero-one programming problem. 

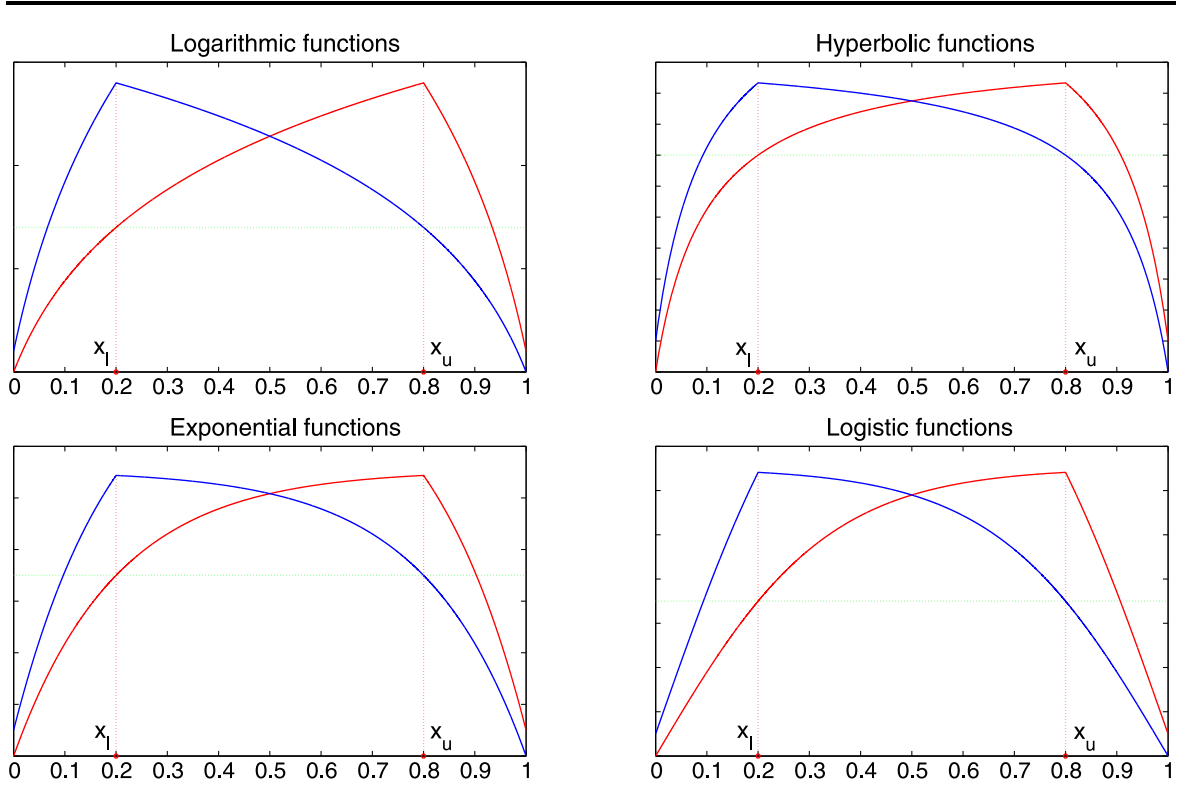

Fig. 2 (Color online) Examples of functions $g_{i}$ for $c_{i}>0$ (in red) and $c_{i}<0$ (in blue). The green line represents $y(x)=\tilde{c}$

As we can easily notice, the functions $g_{i}$ and then the function $f$ depend on the feasible set of the problem. In case $x_{l}=0$, the feasible set does not satisfy Assumption 2.1 and we have that $\phi\left(x_{l}\right)=0$, then we cannot define the continuous reformulation of the original zero-one programming problem. Equivalently, in case $x_{u}=1$, the feasible set does not satisfy Assumption 2.1 and, as $\phi\left(1-x_{u}\right)=0$, we cannot define the continuous reformulation as well. Anyway, when the feasible set is polyhedral, Assumption 2.1 is always satisfied. Then, the class of problems that can be solved by means of our approach includes zero-one linear programming problems.

\section{Conclusions}

In this paper, we described a new continuous reformulation for solving 0-1 programming problems obtained by relaxing the integrality constraints on the variables and by making a nonlinear transformation of the variables in the objective function. We proved that, under suitable assumptions, a given binary problem and its continuous reformulation are equivalent. As we have seen, the class of problems that can be solved by means of our approach includes zero-one linear programming problems. The penalization approach described by Raghavachari in [7] for zero-one linear programming problems has been first extended to the case where the objective function is any concave function [11], then to general nonlinear integer programming problems $[8,15]$. In $[8,15]$, in order to prove the equivalence, the authors only require the feasible set to be compact. Hence a more general class of problems than the one we consider in the paper (including those problems whose feasible set does not satisfy Assumption 2.1) can be solved by using this penalization approach. The method 
described in $[8,15]$ has been further generalized in [12] by showing that a general class of penalty functions, covering the ones proposed in [13], can be used for solving general nonlinear integer programming problems. Also in this case, the authors only assume compactness of the feasible set and a more general class of problems including those ones with a compact feasible set that does not satisfy Assumption 2.1 can be solved. However, we think that the assumption we made on the feasible set might be weakened, and the new approach we described in this paper might be suitably modified to solve a more general class of integer programming problems. Finally, we want to remark that a wider availability of equivalent formulations is important since it can facilitate the search of optimal solutions for different classes of integer problems.

Acknowledgements The authors are grateful to Professor Stefano Lucidi for his useful comments and suggestions. The authors would also like to thank the Referee and the Editor for their insightful comments and the comprehensive and detailed review of the paper.

\section{References}

1. Abello, J., Butenko, S., Pardalos, P.M., Resende, M.: Finding independent sets in a graph using continuous multivariable polynomial formulations. J. Glob. Optim. 21, 111-137 (2001)

2. Balasundaram, B., Butenko, S.: Constructing test functions for global optimization using continuous formulations of graph problems. Optim. Methods Softw. 20, 439-452 (2005)

3. Horst, R., Pardalos, P.M., Thoai, N.V.: Introduction to Global Optimization, 2nd edn. Kluwer, Dordrecht (2000)

4. Mangasarian, O.L.: Knapsack feasibility as an absolute value equation solvable by successive linear programming. Optim. Lett. 3(2), 161-170 (2009)

5. Murray, W., Ng, K.M.: An algorithm for nonlinear optimization problems with binary variables. Comput. Optim. Appl. 47(2), 257-288 (2010)

6. Pardalos, P.M., Prokopyev, O.A., Busygin, S.: Continuous approaches for solving discrete optimization problems. In: Appa, G., Pitsoulis, L., Williams, H.P. (eds.) Handbook on Modelling for Discrete Optimization, vol. 88, pp. 39-60. Springer, New York (2006)

7. Raghavachari, M.: On connections between zero-one integer programming and concave programming under linear constraints. Oper. Res. 17(4), 680-684 (1969)

8. Giannessi, F., Niccolucci, F.: In: Connections Between Nonlinear and Integer Programming Problems. Symposia Mathematica, vol. 19, pp. 161-176. Academic Press, New York (1976)

9. Borchardt, M.: An exact penalty approach for solving a class of minimization problems with Boolean variables. Optimization 19(6), 829-838 (1988)

10. Kalantari, B., Rosen, J.B.: Penalty formulation for zero-one integer equivalent problem. Math. Program. 24, 229-232 (1982)

11. Kalantari, B., Rosen, J.B.: Penalty formulation for zero-one nonlinear programming. Discrete Appl. Math. 16(2), 179-182 (1987)

12. Lucidi, S., Rinaldi, F.: Exact penalty functions for nonlinear integer programming problems. J. Optim. Theory Appl. 145, 479-488 (2010)

13. Rinaldi, F.: New results on the equivalence between zero-one programming and continuous concave programming. Optim. Lett. 3(3), 377-386 (2009)

14. Zhu, W.X.: Penalty parameter for linearly constrained 0-1 quadratic programming. J. Optim. Theory Appl. 116(1), 229-239 (2003)

15. Giannessi, F., Tardella, F.: Connections between nonlinear programming and discrete optimization. In: Du, D.Z., Pardalos, P.M. (eds.) Handbook of Combinatorial Optimization, vol. 1, pp. 149-188. Kluwer Academic, Dordrecht (1998)

16. Rockafellar, T.: Convex Analysis. Princeton University, Princeton (1970)

17. De Santis, M., Lucidi, S., Rinaldi, F.: New concave penalty functions for improving the feasibility pump. Department of Computer and System Sciences Antonio Ruberti Technical Report No. 10, University of Rome "La Sapienza" (2010) 